\title{
Endoscopic Transgastric Necrosectomy for Infected Necrotizing Pancreatitis
}

\author{
O. Joe Hines, MD and Graham W. Donald, MD \\ Department of Surgery, David Geffen School of Medicine at UCLA, Los Angeles, California
}

For decades, treatment of severe acute pancreatitis and pancreatic necrosis incorporated aggressive pancreatic debridement via an open necrosectomy. ${ }^{1}$ The indications for surgical intervention included confirmed or suspected nonviable pancreatic parenchyma, infection within that necrotic collection, or both. This approach often resulted in poor outcomes due to precipitation of a systemic inflammatory response with subsequent organ failure as a result of gross disturbance of an infected necrotic collection. ${ }^{2}$ In the 1990s, it became apparent that this approach was not required for most patients with sterile pancreatic necrosis. ${ }^{3}$ However, patients presenting to the hospital with infected pancreatic necrosis-as evidenced by a positive culture from a pancreatic aspirate or gas in the pancreatic phlegmon identified by CT scan-often were promptly taken to the operating room because of the perception that delay in operative intervention with drainage would result in an extremely high mortality rate. Despite advances in critical care, open necrosectomy has both substantial complication rates and mortality rates, in some series as high as $92 \%$ and $58 \%$, respectively. ${ }^{4,5}$

However, the treatment of necrotizing pancreatitis is rapidly evolving. Numerous studies over the past decade have investigated less-invasive methods for addressing this highly fatal disease process and the less-than-satisfactory historical surgical management. These studies have evaluated procedures ranging from percutaneous drainage of infected necrotic collections, to video-assisted retroperitoneal debridement, to transgastric endoscopic drainage, and various combinations of these modalities. The general consensus is that similar and possibly reduced complication and mortality rates can be achieved by several means other than open necrosectomy.

On the forefront of these efforts are authors from the Dutch Pancreatitis Study Group, who in this issue of $J A M A$ report the results of the PENGUIN (Pancreatitis Endoscopic Transgastric vs Primary Necrosectomy in Patients with Infected Pancreatic Necrosis) trial. ${ }^{6}$ In 2010, this research group reported results of the PANTER (Pancreatitis, Necrosectomy vs Step up approach) trial, a randomized controlled study in which 88 patients with infected pancreatic necrosis were treated with either a "step-up" approach (consisting of percutaneous drainage followed by video-assisted retroperitoneal debridement if necessary) or open necrosectomy. ${ }^{7}$ Although there was no difference in overall mortality between patients in the 2 treatment groups, those in the step-up intervention group had significantly fewer complications, and more importantly, $35 \%$ of patients were adequately treated with percutaneous drainage alone.

(C)2012 American Medical Association. All rights reserved.

Corresponding Author: O. Joe Hines, MD, Department of Surgery, David Geffen School of Medicine at UCLA, 10833 Le Conte Ave, Los Angeles, CA 90095-6904 (joehines@mednet.ucla.edu).

Conflict of Interest Disclosures: All authors have completed and submitted the ICMJE Form for Disclosure of Potential Conflicts of Interest and none were reported. 
In their continued efforts to improve the operative management of patients with infected pancreatic necrosis, Bakker et $\mathrm{al}^{6}$ now report results of a well-designed randomized controlled trial comparing minimally invasive techniques against surgery in 22 patients with infected necrotizing pancreatitis. This trial demonstrates that, compared with surgical debridement, a less invasive approach of natural orifice transluminal endoscopic surgery (NOTES), using transoral endoscopic drainage of infected and necrotic pancreatic collections, significantly reduced the overall inflammatory state and also resulted in lower rates of new-onset multiorgan failure.

The primary clinical end point for this trial was serum levels of IL-6, a surrogate for the overall inflammatory state. ${ }^{8}$ Although the difference in IL- 6 levels between the NOTES group and the surgical group was statistically significant and scientifically compelling, IL-6 has limited utility as a clinical decision-making tool. A clinical composite outcome that included major complications and death also occurred less frequently among patients in the endoscopic group. This group also experienced a lower incidence of pancreatic fistula, which follows given the route of debridement in the 2 groups.

The authors also report that 2 patients who were randomized to the endoscopic group subsequently underwent surgical necrosectomy. Because of the prespecified intention-totreat approach, these patients remained in the endoscopic group in the primary analysis and essentially did not benefit from endoscopic treatment for infected pancreatic necrosis. Additional sensitivity analyses could have been conducted with inclusion of these patients in the surgery group or with eliminating these 2 patients from the analysis. Because of sample size and small numbers of outcome events, these analyses may have attenuated the differences in the composite clinical score, although it seems likely that a significant effect would have remained. The authors are appropriately circumspect in describing their findings as preliminary because the results may have been more robust if the trial recruitment period had been longer and more patients had been enrolled.

The PANTER trial demonstrated the advantages of percutaneous drainage as the initial treatment in patients with infected pancreatic necrosis. This has become the standard surgical approach in many large centers. Data from the report by Rodriguez et al ${ }^{9}$ suggest that it may now be acceptable and preferred to perform drainage procedures earlier and, if possible, to delay more invasive interventions for at least a month. The trial results reported by Bakker et al ${ }^{6}$ imply that endoscopic drainage, even if performed relatively earlier, will lead to fewer complications and less morbidity than open or even video-assisted necrosectomy. Although similar findings have been reported in case series, ${ }^{10}$ larger trials with more robust clinical end points (such as ongoing trials in the Netherlands, ISRCTN09186711) are needed to clearly establish the utility of endoscopic drainage in patients with infected pancreatic necrosis.

\section{References}

1. Traverso LW, Kozarek RA. Pancreatic necrosectomy: definitions and technique. J Gastrointest Surg. 2005; 9(3):436-439. [PubMed: 15749608]

2. Connor S, Alexakis N, Raraty MG, et al. Early and late complications after pancreatic necrosectomy. Surgery. 2005; 137(5):499-505. [PubMed: 15855920]

3. Rau B, Pralle U, Uhl W, Schoenberg MH, Beger HG. Management of sterile necrosis in instances of severe acute pancreatitis. J Am Coll Surg. 1995; 181 (4):279-288. [PubMed: 7551320]

4. Mier J, León EL, Castillo A, Robledo F, Blanco R. Early versus late necrosectomy in severe necrotizing pancreatitis. Am J Surg. 1997; 173(2):71-75. [PubMed: 9074366] 
5. Bone RC. Immunologic dissonance: a continuing evolution in our understanding of the systemic inflammatory response syndrome (SIRS) and the multiple organ dysfunction syndrome (MODS). Ann Intern Med. 1996; 125(8):680-687. [PubMed: 8849154]

6. Bakker OJ, van Santvoort HC, van Brunschot S, et al. Dutch Pancreatitis Study Group. Endoscopic transgastric vs surgical necrosectomy for infected necrotizing pancreatitis: a randomized trial. JAMA. 2012; 307(10):1053-1061. [PubMed: 22416101]

7. van Santvoort HC, Besselink MG, Bakker OJ, et al. Dutch Pancreatitis Study Group. A step-up approach or open necrosectomy for necrotizing pancreatitis. N Engl J Med. 2010; 362(16):14911502. [PubMed: 20410514]

8. Kishimoto T, Akira S, Taga T. Interleukin-6 and its receptor: a paradigm for cytokines. Science. 1992; 258(5082):593-597. [PubMed: 1411569]

9. Rodriguez JR, Razo AO, Targarona J, et al. Debridement and closed packing for sterile or infected necrotizing pancreatitis: insights into indications and outcomes in 167 patients. Ann Surg. 2008; 247(2):294-299. [PubMed: 18216536]

10. Papachristou GI, Takahashi N, Chahal P, Sarr MG, Baron TH. Peroral endoscopic drainage/ debridement of walled-off pancreatic necrosis. Ann Surg. 2007; 245(6):943-951. [PubMed: 17522520] 